\title{
El carácter de la relación entre los factores orgánicos y ambientales en la educación y la enseñanza
}

The nature of the relationship between organic and environmental factors in education and teaching

Dr. Felipe Aguirre Chávez

\section{Resumen}

En la naturaleza humana, los factores biológicos y sociales se funden interactivamente, no es posible estudiarlos por separado. Preguntarse hoy en día si anteponemos lo biológico a lo social o viceversa, resultaría una especie de laberinto cognitivo innecesario como el archiconocido dialelo del "huevo o la gallina".

Lo cierto es que, independiente de los paradigmas o sistemas vigentes en la formación del individuo, tanto, lo biológico como lo social deben atenderse y estudiarse como unidad dialógica, ya que trabajamos con el individuo como persona y como ser social a la vez.

Por tanto, en el proceso de aprendizaje contemporáneo, en que se tiende a mayor libertad de acción y formación con las corrientes desarrolladoras, es necesaria la correlación dialéctica entre lo biológico y lo social.

Palabras clave.- Desarrollo, biológico, social, proceso, educación y enseñanza 


\section{Summary}

In human nature, the biological and social factors are fused interactively, it is not possible to study them separately. To ask oneself today if we put the biological to the social or vice versa, would be a kind of unnecessary cognitive labyrinth like the well-known dialelo of the "egg or the chicken".

The truth is that, regardless of the paradigms or systems in force in the formation of the individual, both the biological and the social must be addressed and studied as a dialogical unit, since we work with the individual as a person and as a social being at the same time.

Therefore, in the contemporary learning process, where there is a tendency to greater freedom of action and training with developing currents, the dialectical correlation between the biological and the social is necessary.

Key words.- Development, biological, social, process, education and teaching 


\section{Introducción}

En el prólogo de su libro La naturaleza humana, Mosterín (2008), indicaba que la naturaleza humana, es decir, lo biológico y social es una cuestión compleja que, eventualmente, sería fácil de responder si se trataría de la pregunta ¿Quién soy yo? Pero, tan difícil porque se quiere saber ¿Qué soy yo?, es decir, ¿En qué consiste la naturaleza humana? y desde el punto de vista pedagógico ¿Cuáles son sus particularidades únicas e irrepetibles y cómo afecta al proceso educativo y de enseñanza aprendizaje?

Históricamente el deseo de comprender la naturaleza humana fue una de las cuestiones más abrumadoras que ha puesto en tensión a los más grandes espíritus de todos los tiempos, condujo a Heráclito de Éfeso afirmar: "Me he buscado a mí mismo" y a Sócrates "Conócete a ti mismo". Cuestión que ha llevado a Ortega y Gaset(1950) deducir que el hombre es un misterio para el propio hombre, probablemente un enigma indescifrable, porque se mire como se mire, está demasiado cerca de sí mismo, está "dentro de él" y al mismo tiempo, "lejos de él mismo". Así, desde los orígenes de la civilización, el tema de la naturaleza humana, sus rasgos esenciales siguen siendo inquietantes. Y, aunque, eventualmente, intentáramos describir, incluso conocer, aun así, seguiría siendo tan difícil de comprenderlo.

En la búsqueda de probables respuestas sobre la correlación entre lo biológico y lo socio-ambiental han primado diferentes consideraciones. Las más influyentes fueron los enfoques unilaterales que plantearon el problema alrededor de tres aspectos desvinculados: lo biológico, lo social y el desarrollo. Jaloneados por un pensamiento fragmentario y reduccionista, la gran mayoría de teóricos se desviaron de su justo cauce y obstruyeron notablemente los esfuerzos por la correcta solución descartando miradas integrales que conciben el desarrollo humano como un proceso complejo de interconexión múltiple en el que se funden aspectos estructurales y funcionales, internos y externos, somáticos y ambientales. Un proceso unísono donde las relaciones ocurren en múltiples direcciones cual ebullición creativa de actos únicos e irrepetibles. Manifestaciones que dejan, muchas veces, en la incertidumbre, sin atisbo de ser explicado y seguirán incomprensibles porque en múltiples casos son impredecibles, originales y multilaterales.

En esa vorágine, la búsqueda de respuestas coherentes, sobre los aspectos biológicos y sociales de la naturaleza humana como factores del desarrollo humano en el proceso educativo, ha constituido una de las constantes preocupaciones de la ciencia y, particularmente, de las ciencias de la educación. Y, aunque la discusión sobre el desarrollo humano, históricamente fue estudiada por la filosofía en la correlación entre lo biológico y lo social, posteriormente es trasladada al área de la psicología y se ha vuelto, ineludiblemente, tema del ámbito de la pedagogía, cuestión que nos motiva ocuparnos en el presente artículo. 


\section{El problema}

Aunque se ha escrito bastante sobre el sentido unitario y multifactorial de la naturaleza humana, sigue vigente y sin resolver, el problema fundamental: ¿Cuál es el carácter de la relación entre los factores orgánicos y ambientales, vale decir, cuáles son los "dominantes" y cuáles los "subordinados"? y de esta gran cuestión derivan otras dos preguntas:

Si, el ser humano está dotado para elaborar representaciones sensoriales y racionales sobre la realidad, sintiendo o actuando dentro de ella para satisfacer sus necesidades, ¿Cuál es la naturaleza de esa actividad psíquica y conductual: biológica o social? Y, más aún, si quedaba establecida la "predominancia" de unos u otros factores, ¿Cuál es el papel de la educación y la enseñanza en la configuración y el desarrollo del ser humano, cómo se debe actuar si la "naturaleza humana" es definida fundamentalmente como biológica o como social? (Merani, 1972).

Estas cuestiones en los hechos indican que el tema de la naturaleza humana sigue siendo tan inquietante como refiere Mosterin (2008): "Entre los fantasmas que ha producido el delirio de la razón, destaca por su extravagancia y recurrencia la idea filosófica de la inexistencia de una naturaleza huumana" (p.23)La naturaleza humana con sus complejidades sigue en el ápice de la filosofía y la ciencia y desde la postura de los educadores se perfila como uno de los más grandes retos pedagógicos de nuestro tiempo. Puede asumirse como factores asimétricos, desvinculados o integrados y en dependencia a ello puede ser de ayuda o de fatal obstáculo en la asunción del desarrollo y resultado del proceso educativo y de enseñanza aprendizaje.

\section{El biogenetismo y sociogenetismo y el desarrollo humano.}

En la explicación de la naturaleza humana en los inicios del siglo XX se consolidaron dos enfoques de carácter polar: el biogenetista y el sociogenetista, aparentemente diferenciados, pero afines desde sus fundamentos.

Desde la perspectiva biogenetista el desarrollo del individuo estaría determinado por aquello que trae consigo con el nacimiento, lo biológico, lo natural y lo herencial. Así, se sobrevalora lo biológico aunque no se niega totalmente el papel "subordinado" de la influencias sociales y entre los elementos biológicos más relevantes destaca la herencia, factor determinante en la configuración de la personalidad y de todas las capacidades. Esta dotación, supuestamente poseedora de gran plasticidad y de innumerables posibilidades de "explicitación", por sí misma, interactuaría con un ambiente inmutable y que sólo sería capaz de "facilitar" o "regular" la manifestación de las potencialidades genéticas. En su modalidad extrema, este enfoque denominado por muchos como "fatalista" se tradujo en el reduccionismo biologista que pretende explicar todos los niveles de la organización y la actividad humana, en particular la psíquica, a partir de factores exclusivamente biológicos, a los que asigna un carácter determinante y decisivo.(Castro, 2005). Más todavía, los biologistas apelando al innatismo plantearon que el leguaje es innato. Por ejemplo, Chomsky (2002) propuso que en el ser humano son innatos los fundamentos biológicos que requiere el lenguaje. En la misma lógica, Jean Piaget (1980) 
defendió que la adquisición del lenguaje se debe a factores biológicos y no culturales. Vale decir, el ser humano llega al mundo con una predisposición, con valores innatos propios de la herencia biológica del cual depende la inteligencia y todos los factores que se desprende de ella.

Desde luego Piaget se distingue de Chomsky cuando indica que el ser humano no posee un lenguaje innato como afirmaba Chomsky (2002), sino que va adquiriéndolo poco a poco como parte del desarrollo cognitivo y una vez adquirido el lenguaje, este a su vez, ayudará también al desarrollo cognitivo.

En el polo opuesto: el sociogenetismo reducía todo lo existente en el ser humano, a simple y mecánico, derivado de las influencias de un medio social ahistórico que actuaría como factor ineluctable o fatal del desarrollo humano, moldearía rígidamente su evolución orgánica y asignaría todas sus características y peculiaridades. Según Castro (2005), para los biologistas el desarrollo del individuo estaría determinado por el ambiente, por sus condiciones de vida y no por su naturaleza orgánica, estas tendrían solo importancia "secundaria". Así, en el estudio del hombre sólo se necesitaría del análisis de la estructura de su ambiente, ya que las particularidades de éste, determinarían de modo irremediable, de una vez y para siempre: la personalidad, los mecanismos de su conducta y las vías del desarrollo psíquico. Al ignorar los rasgos objetivos de éste y al establecer raseros mecánicos para explicar la formación de la personalidad y las capacidades humanas, el sociogenetismo (y su reduccionismo sociologista), nunca han podido aclarar por qué en un mismo medio social se forman personalidades diferenciadas en múltiples aspectos ni por qué existen similitudes psicológicas en individuos formados en entornos socioculturales distintos. Así también, rebajando el psiquismo humano al nivel de elementales y mecánicos "condicionamientos sociales", y subestimando o negando la importancia de los factores biológicos en la constitución del hombre, tanto el sociogenetismo como el sociologismo encontraron expresión psicológica particular en el conductismo y en el neoconductismo y sirvieron de fundamento a la correspondiente pedagogía mecanicista que considera a la educación y a la enseñanza como elementos externos y accesorios en la formación de la personas.

Más aún, todas las particularidades del desarrollo psíquico del individuo, lo mismo que las diferencias individuales, fueron atribuidas a la mayor o menor cantidad de estimulación requerida por el "condicionamiento social" dada para la configuración de una u otra habilidad.

En el plano social, económico y político, históricamente de forma intencionada por los grupos de poder, estos criterios han sido y siguen siendo de utilidad para elaborar justificaciones de desigualdad y exclusión social: los pobres serían personas "insuficientemente condicionadas" y necesitarían "complementar" su cuantía estimulatoria para dejar de ser pobres, siendo la educación y la enseñanza las circunstanciales responsables de cubrir tal "déficit" y de "adaptarlos a la sociedad".

En relación al proceso de enseñanza aprendizaje el sociogentismo y el empirismo moderno, plantearon que la estructura del lenguaje es independiente de las facultades mentales innatas y 
que el ser humano aprende por medio de condicionamientos Skinner (1990), es decir, se reduce el aprendizaje a simples resultados de recompensas y castigos.

En los últimos años, a la histórica dicotomía entre lo biológico y social, al no encontrar, desde sus enfoques sesgados, puntos de encuentro, actualizado la añeja y autocientífica "teoría del paralelismo psicofísico" que, con el nombre de "teoría de los dos factores" (el biológico y el social) en el desarrollo síquico, pretende explicar la evolución humana a partir de la supuesta existencia de las dos líneas paralelas, "determinantes independientes", "subsistemas" o "niveles" en el psiquismo.

En el campo social, basada en la teoría física de las cuerdas separadas, se han ensayado posturas paralelistas que contra lógica intentan explicar que es posible sostener por separado aspectos económicos y políticos. La experiencia indica que eso no es posible por cuanto, los objetos y fenómenos de la realidad como indican Woods y Grant (2010), ocurren en unidad de sus aspectos internos y externos, en simultáneo, entonces es impropio pensar que la línea económica sea opuesta o independiente de la líneas política como los aspectos internos de los externos, por lo mismo, que lo material de lo espiritual. Por el contrario, son factores inseparables como ocurre entre el desarrollo y educación el hombre y la sociedad.

\section{El papel de la herencia y de la acción de los factores socio-ambientales.}

Estudios realizadas con gemelos, particularmente con los univitelinos, es decir, monocigóticos o uniovulares (originados por la fecundación de un solo óvulo por un único espermatozoide, con el resultado de la división de ese óvulo fecundado en dos células con la misma dotación genética, las que conducirán a la formación de dos individuos físicamente idénticos). Esas investigaciones, sobre todo las efectuadas por René Zazzo, demuestran que las peculiaridades de tales gemelos, especialmente las psíquicas, son originadas por el entrecruzamiento de factores internos y externos que interactúan y se condicionan recíprocamente, actuando los segundos a través del estado específico de los primeros y teniendo además, como mediación esencial, la actividad integral del sujeto para generar las particularidades que distinguen a una persona de todas las demás, resultando así absurdo plantear "qué porcentaje" aporta cada tipo de factores. Ocurre que "dos gemelos, poseedores del mismo potencial genético, pero colocados ante influencias socioculturales distintas, tendrán personalidades diferentes, e incluso en las mismas condiciones ambientales elaborarán cada cual rasgos peculiares y diferenciales. En uno u otro caso, existen diferencias en la disposición de las condiciones internas de cada persona, de modo que los factores externos actuarán sobre estados distintos de las mismas y siempre tendrá valor decisivo un aspecto fundamental: el carácter mediador de la actividad, que en definitiva, es el elemento básico para la formación y el desarrollo de los rasgos y particularidades que definen a un individuo como tal" (Castro, 2005).

Por otra parte, las peculiaridades biológicas del sistema nervioso y de otros sistemas del organismo humano, constituyen la base anátomo-fisiológica de lo que Vigotsky (1979), denominaba también "fuerzas vitales que el hombre posee parcialmente de nacimiento y que 
existen en él en forma de premisas y capacidades potenciales". Sin embargo, tales premisas no son cualidades físicas y psíquicas ya "preparadas" y listas para manifestarse, sino, elementos potenciales naturales para la formación y el desarrollo de tales cualidades (las cuales pueden convertirse en realidad o frustrarse según las particularidades del tipo de circunstancias sociales específicas que actúan sobre las mencionadas premisas individuales). En su conjunto, las premisas representan la potencialidad humana en general con unas u otras peculiaridades tipológicas personales; constituyen el resultado de las primeras etapas recorridas por cada individuo en su desarrollo en determinadas condiciones internas y externas y, como tales, son fenotipos cuyo vínculo con las condiciones internas de partida en el desarrollo puede tener diferente significado y conducir a resultados distintos de un individuo a otro según el tipo de influencias ambientales a las que ellos están expuestos. Por ejemplo, se sabe con certeza que la desnutrición crónica en los primeros años de vida, que afecta a gran número de niños en amplios sectores populares flagelados por la pobreza y la miseria, anula esencialmente las posibilidades de desarrollo contenidas en una dotación genética considerada como adecuada; y, a la inversa, una correcta nutrición en un ambiente propicio es uno de los elementos favorables para el encauzamiento y despliegue de las potencialidades existentes. Tampoco posee un mismo significado el vínculo de las premisas dadas con las futuras propiedades psíquicas de la personalidad sobre la base de esas premisas, y en el curso de la interacción del individuo con el medio social y natural, pueden desarrollarse y se desarrollan diversas cualidades psíquicas de la personalidad con características particulares y diferenciales para cada sujeto (en concordancia con la edad, las condiciones concretas de existencia, la pertenencia social de clase, la estructura del hogar y la personalidad de los padres, la actividad y las vivencias del sujeto, las relaciones interpersonales y de grupo, la estimulación sociocultural asimilada, la influencia de la educación y la enseñanza, etc.). De tal suerte, las premisas son indispensables, pero al mismo tiempo insuficientes para la formación y desarrollo de las cualidades; es decir, constituyen condiciones internas favorables para la asimilación de la experiencia social e influyen en la dinámica de la elaboración de los nuevos mecanismos de la conducta y de las propiedades psíquicas que en ellos se manifiestan, pero no determinan por sí mismas su contenido y su orientación, las cuales son aportados por las diversas influencias del medio socio-cultural en el que tiene lugar la vida y la actividad del sujeto.

Estos aspectos fundamentales, poseedores de una vasta comprobación científica, necesitan ser tenidos en cuenta por la educación y la enseñanza), para apreciar al ser humano como ser social singularizado en sus expresiones individuales; como unidad dialéctica de lo orgánico y lo social que perfila su especificidad personal a través de la comunicación y la interacción con las otras personas y en el curso de su propia actividad; y cuyo desarrollo debe ser afrontado de manera integral, es decir, encarado para lograr la formación y el despliegue simultáneo e inseparable de su personalidad y sus capacidades físicas, psíquicas y morales. Por ello, es necesario también considerar en su interacción dialéctica las condiciones internas y externas del desarrollo humano. 


\section{Perspectivas de integración de enfoques sobre el desarrollo humano}

Las perspectivas sobre la naturaleza humana, del carácter de lo biológico y social, en el proceso educativo y de enseñanza aprendizaje en el presente artículo se realiza bajo la estela del desarrollo humano que en la actualidad presenta perspectivas integradoras y dinámicas aunque no por ello dejan de estar comprometidas. En un intento de superación de las tesis rancias sobre el desarrollo humano el Programa de las Naciones Unidas para el Desarrollo (PND) plantea que, es un proceso y resultado que busca que las personas puedan desarrollar su máximo potencial y llevar adelante una vida productiva y creativa de acuerdo con sus necesidades e intereses donde el talento humano constituye el mayor capital de los pueblos y que su desarrollo debe ser impostergable. Otra definición interesante sobre el desarrollo humano aparece en el Informe del Club de Roma (1972) citado por Martínez, Lanuez y Llantada (2002), quienes consideran:

El desarrollo como el proceso que experimenta una sociedad para conseguir el bienestar de la población, relacionándose de forma armónica con el entorno natural, consiguiendo así satisfacer las necesidades materiales y establecer las bases para que todo individuo pueda desplegar su potencial humano. (p. 5)

La centralidad humana del desarrollo en sus múltiples variantes no solo busca garantizar el bienestar material y espiritual, sino el de posibilitar el logro de la felicidad. Esto es el rasgo novedoso del nuevo modelo de desarrollo humano que remplaza a aquellos que solo se centraban en lo económico, lo cognitivo solamente, o focalizado en aspectos estrictamente materiales.

El nuevo desarrollo humano revolucionario plantea el perfeccionamiento integral e interactivo. Un desarrollo vivo que en la perspectiva de Castro (2005), tiene implicancias con las funciones psíquicas superiores cuyo desarrollo, solo es posible a través de la educación, (herramienta y valor para lograr mejores niveles de intercambio y relación con la naturaleza y con la sociedad), de participación en la vida ciudadana, de configuración de estrategias para lograr un hombre creativo, capaz de enfrentarse a los múltiples problemas de diferente índole y buscar soluciones novedosas a los problemas cotidianos.

Lanuez, Martínez y Llantada (2002) refieren que la educación es un satisfactor de necesidades humanas, y para lograr un desarrollo humano sostenible, la educación como proceso educativo y de enseñanza aprendizaje lo prepara con el desarrollo de sus capacidades que le permitan participar en el desarrollo y en la actividad social. Así, la educación constituye condición para el desarrollo total del hombre, y a su vez es resultado de esa condición. Algo que Maus citado por Herrera (1957) explicó asertivamente: "la educación tiene que ver el con el hombre y su vida en sociedad, en la consideración de tres dimensiones irreductibles e inseparables del individuo: el cuerpo, la conciencia individual y la conciencia colectiva" (p.12).

El hombre en su sentido total es la simbiosis de aspectos internos y externos, de elementos físicos y espirituales. Satisfacer sus necesidades, probablemente solo sea posible desde enfoques integrales como el del desarrollo sostenible que al decir de Valencia (2016) "La única manera de superar esto es teniendo un entendimiento del bienestar social" (p. 16) que se encuentra 
ligada al bienestar del desarrollo de la habilidad mental y en función a la mejora de la calidad de vida, aumentaría el desarrollo del potencial desarrollador, proactivo y de búsqueda de mejoras integrales. Esto según la Organización Mundial de la Salud,(2012) recibe el nombre de "bienestar intelectual" que constituye la capacidad de mantener relaciones armoniosas con los demás, y al mismo tiempo de resolver conflictos sin herir a las otras personas.

Un factor central en el bienestar intelectual es el desarrollo de la habilidad mental. En función a la mejora de la calidad de vida, aumenta el desarrollo del potencial intelectual desarrollador, proactivo y de búsqueda de mejoras integrales.

Superando amplia y categóricamente las posturas unilaterales, el enfoque integrador de la correlación entre lo biológico y lo social establece que la naturaleza humana no es algo abstracto e inmutable, sino un concreto y cambiante resultado del desarrollo socio-histórico; el hombre modifica su propia naturaleza en el curso del proceso de transformación del mundo socionatural para adecuarlo a la satisfacción de sus diversas necesidades, Vygotsky (2001).

En el desarrollo humano el hombre es protagonista de un proceso único en el que intervienen tanto los principios biológicos y sociales donde el reconocimiento del papel decisivo de las condiciones sociales presupone la existencia de las premisas orgánicas como absolutamente necesarias. Lo biológico y lo social al conformar una unidad dialéctica de opuestos complementarios no deja de estar mediatizado por sus condiciones sociales de existencia y, simultáneamente, por sus dotaciones genéticas.

\section{La educación como medio de desarrollo humano}

El elemento clave para que las personas logren el desarrollo, tengan calidad de vida, es la educación. Sin ella, las personas no podrán participar de los adelantos del mundo ni podrán acceder al trabajo dignamente reconocido.

La educación en esa perspectiva constituye un satisfactor de necesidades humanas, y para lograr el desarrollo humano integral prepara al hombre, estimula y coadyuva al desarrollo de sus capacidades, lo provee de conocimientos, que le permiten participar en el desarrollo y en la actividad social en general, pero además, el desarrollo a su vez constituye un medio para mejorar la calidad de vida, y la educación propende también el aumento de dicha calidad; por todo ello, la educación constituye una condición para el desarrollo, y a su vez es su resultado; de ahí su lugar clave en el desarrollo humano.

Por eso es muy importante la educación, que como proceso y resultado puede ser asumida en su sentido de herramienta de desarrollo o de opresión. Cualquier postura frente a la educación dependerá del enfoque desde el que se asume. El enfoque es determinante.

En esa lógica a la pregunta ¿qué entendemos por educación? podríamos afirmar que es esencialmente un proceso intencional de carácter teleológico; de no ser así sería un proceso sin norte, sin fines, que no propone llegar a ninguna parte. El pedagogo peruano Walter Peñaloza (2003) graficó el sentido de la educación al afirmar que: "No educamos al azar, para 
sorprendernos al final al ver que ha sucedió, sino que lo hacemos con una determinada idea de lo que anhelamos" (p.27) a estos propósitos, que guían nuestras acciones los llamamos finalidades o también objetivos. No existe educación espontánea o improvisada. La educación como proceso y resultado es un acto intencional de formación y de desarrollo armónico de la personalidad desde el momento mismo de su nacimiento y durante todo el curso de su existencia que según José Martí (1990, p.281) "...es hacer a cada hombre resumen del mundo viviente... preparar al hombre para la vida". La consecución de tal finalidad no puede tener lugar de manera inmediata, sino a través de un proceso prolongado marcado por una especie de hitos o peldaños concretos denominados objetivos educacionales, cuyos logros sucesivos en el curso de la actividad escolar y pedagógica conducen a alcanzar el fin propuesto. En palabras de Labarrere y Valdivia (2003):

De la fijación de los objetivos se derivan las tareas que contribuyen a la formación multilateral del individuo, considerada no como una simple adición o suma aritmética de los distintos componentes, sino como una integración armónica de estos factores en la personalidad del educando.(p.35).

La educación como proceso y resultado es un acto mediado por los aspectos biológicos y sociales que siempre es necesario tener en cuenta así para apreciarlo objetivamente. Existe infinidad de factores que la afectan, por ejemplo los aspectos físico-orgánicos (condiciones materiales de vida y actividad, estado nutricional y de salud del estudiante), psicopedagógicos (estadio del desarrollo y particularidades psicológicas del escolar, estado y nivel de sus conocimientos previos, estilos y ritmos cognitivos, motivación y auto-motivación técnicas de estudio), pedagógico-metodológicos (concepción del mundo, características de la personalidad, nivel científico-profesional, estilos y métodos de enseñanza del docente) y, naturalmente, las condiciones concretas en las que se desenvuelve el proceso educativo (ambiente escolar, números de estudiantes por aula, infraestructura educacional, medios y materiales didácticos). Sin precisar adecuadamente las particularidades concretas de cada uno de estos elementos y su entrecruzamiento recíproco, se puede derivar con facilidad hacia una visión formalista y abstractizada del proceso de enseñanza aprendizaje, ignorado las realidades y cargando a la cuenta del docente o del educando (o de ambos) los resultados insatisfactorio del proceso educativo y enseñanza-aprendizaje.

En todo caso, como decía Aníbal Ponce (1975):

Cada uno de nosotros construye su personalidad no sólo sobre la base que le da el temperamento, sino también sobre los aportes que le da una educación impuesta por el medio familiar como un reflejo, a su vez, del ambiente social en que nos desenvolvemos. Este medio social no es homogéneo y la educación no es idéntica para todos los miembros. La sociedad, por el contrario, está dividida en clases, con intereses e ideales irreconciliables; y en cada momento de la evolución histórica son las ideas de la clase dominante las que se erigen en las ideas llamadas de la época (p.36) 
Por consiguiente, en la formación de las personas, en la configuración y el perfilamiento de su conciencia, pensamiento, actitudes, sentimientos y prácticas según los requerimientos del sistema de producción dado (que, a su vez, se corresponden con las necesidades e intereses de la clase dominante en él), la educación y la enseñanza cumplen un rol decisivo para el mantenimiento indefinido del estado de cosas vigente o puede servir como medio de evaluación crítica promoviendo la transformación social. Según sea su carácter, contenido, orientación y proyecciones (es decir, de acuerdo con el tipo de concepción del mundo y de ideología que les dan cuerpo y las ponen en acción), la educación y la enseñanza constituyen agentes de apaciguamiento y conformismo, o vectores dinámicos del cambio esencial en la vida de la sociedad. Por ello, desde una perspectiva objetiva y científica, la educación y la enseñanza siempre poseen un sello, están al servicio de los intereses de una u otra clase, propugnan el quietismo y el estancamiento o la transformación activa de la sociedad, y nunca existen en forma "neutra", "independiente", "pura", ajena a la realidad social y sus contradicciones y conflictos concretos que en ella tienen lugar, Aníbal Ponce (1975).

\section{El rol del maestro}

Uno de los factores más importantes en el proceso educativo y de enseñanza aprendizaje del desarrollo humano es la figura del maestro. La forma y estilo de cómo se desarrolla la vinculación maestro-estudiante son determinantes en el proceso y resultado de logros de aprendizaje. El docente por su concepción del mundo o su formación profesional podría asumirse como orientador y transformador de conciencias o un facilitador mecánico. Como orientador dirigirá el proceso de enseñanza-aprendizaje, proyectará y ejecutará la formación y el desarrollo de capacidades, convicciones que constituyen reguladores fundamentales de actuación y comportamiento del estudiante en la vida social.

Bajos estas premisas en el terreno pedagógico la orientación es el opuesto concreto y racional de la subjetiva "facilitación". Orientar significa dirigir y promover el proceso de enseñanzaaprendizaje. En cambio "facilitar" consiste en propiciar las condiciones para que el estudiante exprese sus potencialidades. Esto que es aparentemente bueno en esencia reduce el rol del maestro a un rol de subordinado, es decir, desconoce el carácter de la relación simétrica e interactiva entre el docente y el estudiante en el proceso de enseñanza aprendizaje.

En efecto, entre orientación y facilitación se evidencia dos concepciones antagónicas del proceso educativo y de enseñanza aprendizaje. La orientación asume el carácter social de las capacidades, mientras que la facilitación supone su innatismo. Según Castro (2005) desde el punto de vista científico y con toda justicia el Maestro en el nivel que trabaje cumple los roles de "orientador" y "transformador". En su función transformadora yace determinada la delicada tarea de promover estimular y dirigir la evolución integral del estudiante y a su vez le prepara para el encaramiento objetivo y ecuánime de las circunstancias y problemas que plantea el discurrir vital. Así, también define el progresivo desarrollo de sus capacidades y actitudes para definir una opción social-laboral. 
En suma, el desarrollo adecuado de la tarea orientacional contribuirá significativamente en el desarrollo humano, es decir, en la configuración del hombre total con rasgos democráticos, de equidad, calidad de vida e integración social. Por cuanto, el nuevo tipo de hombre será el núcleo de desarrollo de las familias y, a la postre, de la sociedad en perspectiva.

\section{Conclusiones}

Primero.- Podemos afirmar que tanto uno como otro factor, no podemos estudiarlos por separados sin la debida interdependencia.

Segundo.- El preguntarse hoy en día si anteponemos lo biológico a lo social o viceversa durante el proceso docente educativo, resultaría una especie de laberinto cognitivo innecesario como el archiconocido caso del "huevo o la gallina".

Tercero.- Lo cierto es que, independiente de los paradigmas o sistemas vigentes en la formación del individuo, tanto, lo biológico como lo social deben atenderse y estudiarse como una unidad dialógica, ya que trabajamos con el individuo como persona y como ser social a la vez.

Cuarto.,- En el proceso de aprendizaje contemporáneo, en que se tiende a mayor libertad de acción y formación con las corrientes constructivistas, es absolutamente necesaria esta correlación entre lo biológico y lo social.

Quinto.- Uno de los factores más importantes en el proceso educativo y de enseñanza aprendizaje del desarrollo humano es la figura del maestro. La forma y estilo de cómo se desarrolla la vinculación maestro-estudiante son determinantes en el proceso y resultado de logros de aprendizaje. 


\section{REFERENCIAS}

Aguirre, F (2007) "Formación de capacidades" Lima: EDUCAP. (2006) "El Proceso de enseñanza y aprendizaje: tarea docente y actividad del educando" Lima: EDUCAP.

Castro, L. Aguirre, F. Taboada, M. y Gastañaduy, R. (2007) "Formación Psicopedagógica del Docente" Perú: Epla.

Castro, L. (2007) "Elementos de psicopedagogía docente" (2da.Ed) Lima: EDUCAP. (2005) "Diccionario de ciencias de la educación" (2da.Ed) Lima: EDUCAP.

Castro y otros (2006) "Módulo introductoria de psicopedagogía" Lima: EDUCAP.

Fabelo, R, Jesús P. y otros (2006) "Educación, ética y desarrollo integral del educando" (1 ra Ed.) Perú: EDUCAP.

Ferrater, J. (1991) "Diccionario de Filosofía". Madrid: Aula Santillana, S.A.

Hayek, F. (1944) "Camino de servidumbre" Madrid: Alianza.

Gramsci, A (1967) "La formación de los intelectuales" México: Colección 70, Editorial Grijalbo.

Lanuez, Martínez y Llantada (2002) La investigación científica en el siglo XXI.Ed- EL Pueblo. Cuba

Petrovski, A. (1979) "Psicología General" Ciudad de la Habana: Editorial Pueblo y Educación.

Ponce, Al (1970) "Educación y lucha de clases" Buenos Aires: Editorial El viento en el mundo. 96. (1975) "Humanismo burgués y humanismo proletario" Buenos Aires - Argentina: Editorial Cartago.

Smirnov, Leontiev, Rubinstein y Tieplov (1960) "Psicología" (1ero.ed) México: Grijalbo.

Mosterin, J (2008) La naturaleza Humana. Madrid: Espasa Calpe.

Vigotsky, L (2008) "Imaginación y creación en la edad infantil" Perú: EDUCAP. (1996)"El desarrollo de los procesos psicológicos superiores" (1 ra.Ed) Barcelona: Grijalbo Mondadori.

Woods, A. y Grant, T (2002) "Razón y revolución" (2da.Ed) España: Fundación Federico Engels.

*AGUIRRE CHAVEZ, FELIPE. Master y Doctor en educación. Desde el 2005 ejerce la docencia en las Escuelas de Posgrado de las universidades: Universidad Nacional de Educación, San Ignacio de Loyola y Marcelino Champagnat. Trabajó como especialista en el Ministerio de Educación del Perú y participó como auditor educativo en la Contraloría General de la República. Autor de textos, artículos científicos y de proyectos de innovación que han recibido premios nacionales (2002, 2004,2007 y 2012). 